\title{
Anti-inflammatory Activity in the Aqueous Crude Extract of the Leaves of Nidularium procerum: A Bromeliaceae from the Brazilian Coastal Rain Forest
}

\author{
Fabio Coelho Amendoeira, ${ }^{a}$ Valber Silva Frutuoso, ${ }^{a}$ Cristiane Zanon, ${ }^{a}$ Luciana Moreira Chedier, ${ }^{b}$ \\ Maria Raquel Figueiredo, ${ }^{b}$ Maria Auxiliadora Coelho Kaplan ${ }^{c}{ }^{c}$ Christianne Bandeira-Melo, ${ }^{a}$ \\ Patrícia Torres Bozza, ${ }^{a}$ and Hugo Caire CASTRo-Faria-Neto ${ }^{*, a}$ \\ ${ }^{a}$ Laboratório de Imunofarmacologia, Departamento de Fisiologia e Farmacodinâmica e; Fundação Oswaldo Cruz, Av. \\ Brasil 4365, Rio de janeiro, RJ, 21045-900, Brazil: ${ }^{b}$ Laboratório de Produtos Naturais 3 (PN-3) Manguinhos; Fundação \\ Oswaldo Cruz, Rio de Janeiro, Brazil: and ${ }^{c}$ Núcleo de pesquisas em produtos naturais (NPPN/UFRJ); Rio de Janeiro, \\ Brazil. Received October 26, 2004; accepted February 18, 2005
}

Nidularium procerum Lindman, a common bromeliaceae from the Brazilian flora, remains poorly studied regarding its chemical and pharmacological properties. We have recently published that $N$. procerum has potent analgesic and anti-inflammatory activities. In the present work, we have investigated potential mechanisms involved in the anti-inflammatory effects of $N$. procerum aqueous extract on lipopolysaccharide (LPS)-, platelet activating factor (PAF)- or formyl-methionyl-leucyl-phenylalanine (fMLP)-induced pleurisy models of inflammation. We found that the aqueous extract of $N$. procerum leaves (leaf aqueous extract; LAE) inhibits the neutrophil migration, production of inflammatory cytokines interleukin-1 and -6 (IL-1 and IL-6) and the generation of prostaglandin $\mathrm{E}_{2}\left(\mathrm{PGE}_{2}\right)$ in LPS-induced pleural inflammation in mice. Such inhibitory effect of $N$. procerum on $\mathrm{PGE}_{2}$ generation was tightly correlated to the inhibition of formation of new cytoplasmic lipid bodies within recruited leukocytes. $N$. procerum also blocked the in vivo neutrophil influx induced by injection of PAF or fMLP into the mouse pleural cavity and directly inhibited PAF-induced neutrophil chemotaxis in vitro. The data obtained in this study indicate that $N$. procerum LAE exerts its anti-inflammatory effects by interfering with the capacity of the host to respond to injury at different levels. Among the different functions affected by $N$. procerum LAE, lipid body formation, $\mathrm{PGE}_{2}$ and cytokine production and neutrophil chemotaxis are readily evidenced in relevant surrogate models. The $N$. procerum bioactive profile makes it an attractive candidate for future development as a drug or phytomedicine.

Key words Nidularium procerum; anti-inflammatory activity; natural product; chemotaxis; neutrophil

Brazilian flora, especially in the Brazilian coastal rain forest, remains poorly studied regarding its chemical and pharmacological potential. A recent study published by our group indicates that Nidularium procerum, the most common representative of the bromeliaceae family, has potent analgesic and anti-inflammatory activities. ${ }^{1)}$ Bromeliaceae, one of the largest botanical families of the New World, is extensively distributed in tropical America. ${ }^{2)}$ This family comprises 46 genera encompassing about 2500 species from which 20 genera and 245 species are found in the State of Rio de Janeiro. The Brazilian coastal rain forest is the preferred habitat of Bromeliaceae $(81.8 \%)$ where the greatest genetic diversity and the highest degree of endemism is found. ${ }^{3)}$ Species of Nidularium are well spread in the coastal rain forest from Bahia State, in the northeast region of Brazil, to Rio Grande do Sul State in the south. ${ }^{4)}$

Our previous results indicate that $N$. procerum extracts have strong analgesic activity, which is not associated with the activation of opioid receptors, but is mechanistically due to a selective inhibition of $\mathrm{PGE}_{2}$ production. ${ }^{1)}$ In addition, we showed that the aqueous extract of $N$. procerum leaves (LAE) inhibited LPS-induced neutrophil accumulation in the mouse pleural cavity, but the molecular mechanism for this anti-inflammatory effect was not investigated. ${ }^{1)}$ Here, we investigated the mechanisms involved in the anti-inflammatory effects of $N$. procerum aqueous extract on the LPS-induced pleurisy model. We showed that in addition to LPS-induced neutrophil influx, $N$. procerum also inhibited LPS-driven in vivo neutrophil activation (as attested by inhibition of lipid body formation) and PAF- and fMLP-induced pleural neutrophilia. The mechanisms involved in N. procerum-mediated inhibition of neutrophil recruitment appear to involve decreased production of key inflammatory mediators of LPSdriven response, such as $\mathrm{PGE}_{2}$ and inflammatory cytokines (IL-1 and IL-6), and an impaired locomotory activity of migrating neutrophils.

\section{MATERIALS AND METHODS}

Plant Material Nidularium procerum Lindman was collected at the National Biological Reserve of Poço das Antas, Silva Jardim, Rio de Janeiro State, Brazil. Identification of the botanical material was made by Dr. Tânia Wendt and Dr. Elton Leme and a voucher has been deposited at the Herbarium of the Rio de Janeiro Botanical Garden, under the number 304732 .

Preparation of Plant Extracts The aqueous extract of leaves (LAE) (22 g) was obtained by infusing dried leaves $(500 \mathrm{~g})$ into 2.51 of boiling distilled water until the water cooled down to room temperature, followed by filtration and lyophilization as previously described. ${ }^{1)}$

Animals Male Swiss mice (obtained from the Oswaldo Cruz Foundation breeding unit) weighing $20-30 \mathrm{~g}$, were used. The animals were maintained with free access to food and water and kept at $25-28^{\circ} \mathrm{C}$ with controlled $12 \mathrm{~h}$ light/dark cycle at the Department of Physiology and Pharmacodynamics. The experiments in this study received prior approval from the Oswaldo Cruz Institute's Animal Welfare 
Committee.

Pleurisy Pleurisy was induced by intrathoracic (i.t.) injection of different stimuli, and the control group was injected with vehicle (sterile saline). Treatments were administered $1 \mathrm{~h}$ before stimulus injection. Six hours after i.t. challenge the animals were killed in a $\mathrm{CO}_{2}$ chamber and the thoracic cavity was opened and washed with $1 \mathrm{ml}$ phosphate buffered saline (PBS). The pleural wash was collected with a pipette and used for analysis of the cellular infiltrate (total and differential leukocyte counts), $\mathrm{PGE}_{2}$ and cytokine levels. The total number of leukocytes collected in the pleural wash was evaluated in a Neubauer chamber after dilution of a pleural wash sample in $2 \%$ acetic acid. The differential analysis of leukocytes was accomplished in cytosmears stained with May-Grunwald-Giemsa dye.

Treatments and Stimuli Intrathoracic challenge was performed with lipopolysaccharide (LPS; $250 \mathrm{ng} /$ cavity), formyl-methionyl-leucyl-phenylalanine (fMLP; $200 \mathrm{ng} / \mathrm{cav}-$ ity) ${ }^{6)}$ or platelet activating factor (PAF; $500 \mathrm{ng} /$ cavity) ${ }^{7)} 1 \mathrm{~h}$ after treatment. Treatments consisted of an intraperitoneal (i.p.) injection of LAE of $N$. procerum $(0.2,2,20 \mathrm{mg} / \mathrm{kg}$ i.p.) or acetyl salicylic acid (ASA; 100, 200, $500 \mathrm{mg} / \mathrm{kg}$ ). All treatments were diluted in sterile saline immediately before use.

Lipid Body Staining and Enumeration To enumerate the lipid bodies within leukocytes, the pleural wash cytosmears were fixed in 3.7\% formaldehyde diluted in Hank's Balanced Salt Solution $\left(\mathrm{Ca}^{2+} / \mathrm{Mg}^{2+}\right.$-free HBSS; $\left.\mathrm{pH} 7.4\right)$, rinsed in $0.1 \mathrm{M}$ cacodylate buffer ( $\mathrm{pH} 7.4$ ), stained with $1.5 \% \mathrm{OsO}_{4}$ for $30 \mathrm{~min}$, rinsed in distilled $\mathrm{H}_{2} \mathrm{O}$, immersed in $1.0 \%$ thiocarbohydrazide for $5 \mathrm{~min}$, rinsed in $0.1 \mathrm{M}$ cadodylate buffer, re-stained with $1.5 \% \mathrm{OsO}_{4}$ for $3 \mathrm{~min}$, rinsed in distilled water and then dried and mounted as previously described. ${ }^{8}$ Cell morphology was observed and lipid bodies were enumerated by microscope under an oil immersion objective $(100 \times$ magnification). Twenty-five consecutively scanned leukocytes were evaluated and results were expressed as the number of lipid bodies per leukocyte.

Measurements of Pleural Fluid $\mathbf{P G E}_{2}$ Pleural fluid Prostaglandin $\mathrm{E}_{2}\left(\mathrm{PGE}_{2}\right)$ concentration in the cell supernatant was assayed in duplicate by enzymatic immunoassay (EIA) according to the manufacturer's instructions (Cayman, Ann Arbor, MI, U.S.A.).

Murine Macrophage Cultures Mice were intraperitoneally injected with $2 \mathrm{ml}$ of $3 \%$ thioglycollate. On day 3, the peritoneal cells were obtained by peritoneal lavage with $5 \mathrm{ml}$ of HBSS, $\mathrm{Ca}^{2+}$ and $\mathrm{Mg}^{2+}$ free. These cells were washed twice and resuspended in Roswell Park Memorial Institute1640 medium (RPMI-1640) supplemented with 10\% fetal calf serum, $100 \mathrm{U} / \mathrm{ml}$ penicillin and $100 \mu \mathrm{g} / \mathrm{ml}$ streptomycin. Cells were plated at a density of $1 \times 10^{6}$ cells/well in a 24 well culture plate (Falcon Labware, Lincoln Park, NJ, U.S.A.). Plates were incubated in a humidified atmosphere containing $5 \% \quad \mathrm{CO}_{2}$ at $37^{\circ} \mathrm{C}$ for at least $1 \mathrm{~h}$ to allow macrophage adherence Then were washed by gentle agitation with $\mathrm{HBSS} \mathrm{Ca}^{2+}$ and $\mathrm{Mg}^{2+}$ free and RPMI-1640 medium was added to the wells.

Induction of Cytokine Release by Macrophages in Vitro Murine resident peritoneal macrophages $\left(10^{6}\right.$ cells $/ \mathrm{ml}$ in RPMI-1640) were pretreated with $N$. procerum LAE (2 or $0.2 \mu \mathrm{g} / \mathrm{ml}$ ) for $15 \mathrm{~min}$ and then stimulated with LPS (500 ng/ $\mathrm{ml}$ ) for $4 \mathrm{~h}$. Cells were centrifuged and cytokines released in the supernatant were measured as described below.

In Vitro Induction of Lipid Body Formation withi Murine Macrophages Murine resident peritoneal macrophages $\left(10^{6}\right.$ cells $/ \mathrm{ml}$ in HBSS $)$ recovered from the peritoneal cavity of naive mice were stimulated with PAF $(1 \mu \mathrm{M})$, LPS $(500 \mathrm{ng} / \mathrm{ml})$ or vehicle $(\mathrm{HBSS})$ at $37^{\circ} \mathrm{C}$ for $1 \mathrm{~h}$. The leukocytes were pretreated with $N$. procerum LAE ( 2 or $0.2 \mu \mathrm{g} / \mathrm{ml}$ ) for $15 \mathrm{~min}$ before stimulation. After stimulation, cells were prepared for lipid body evaluation, as described above.

Measurements of Pleural Fluid Cytokines Cell free supernatants were used for determination of IL- $1 \beta, \mathrm{KC}$, IL10 and IL-6 levels within pleural fluid by ELISA using commercially available kits and following the instructions provided by the manufacturer (Duo set kit; R\&D Systems, Mineapolis, MN, U.S.A.).

Human Neutrophil Purification Neutrophils were purified from fresh human blood obtained by venipuncture from healthy adult volunteers and collected in acidified citrate. After addition of $6 \%$ dextran 70 (McGaw, Irvine, CA, U.S.A.), red blood cells (RBC) were allowed to sediment for $1 \mathrm{~h}$ at room temperature. The leukocyte-rich supernatant was overlaid onto an equal volume of Ficoll-Paque gradient (Pharmacia, Piscataway, NJ, U.S.A.), and centrifuged at $400 \boldsymbol{g}$ for $20 \mathrm{~min}$. Polymorphonuclear leukocytes (PMN; 95\% pure, with eosinophils as main contaminants) were recovered from the pellet and washed in $\mathrm{Ca}^{2+} / \mathrm{Mg}^{2+}$-free HBSS. Residual RBCs were lysed with hypotonic saline.9)

Human Neutrophil Chemotaxis Assay Migration experiments were performed using a 48-well microchemotaxis chamber (Neuro Probe, U.S.A.) and Toyo cellulose nitrate filters $\left(3 \mu \mathrm{m}\right.$ pore), as described previously. ${ }^{10,11)}$ PAF $\left(10^{-6} \mathrm{M}\right)$ and RPMI-1640 medium containing bovine serum albumin $(29 \mu \mathrm{l})$ were placed in the lower chamber compartment and $50 \mu \mathrm{l}$ of the human neutrophil suspension $\left(2.5 \times 10^{5}\right.$ cells/ $50 \mu \mathrm{l})$ was placed in the upper chamber compartment. Purified human neutrophils were pre-incubated with $N$. procerum LAE $(0.01 \mathrm{mg} / \mathrm{ml})$ or culture medium at $37^{\circ} \mathrm{C}$ in a $5 \% \mathrm{CO}_{2}$ atmosphere. Fifteen minutes after the pre-incubation period, the cells were placed in the chamber and incubated for $2 \mathrm{~h}$ at $37^{\circ} \mathrm{C}$ in a $5 \% \mathrm{CO}_{2}$ atmosphere. The filters were fixed and stained as described. ${ }^{10)}$ Human neutrophils found at $40 \mu \mathrm{m}$ from the upper surface of the filter were counted in 15 consecutive high power fields (HPF).

In Vitro Induction of Lipid Body Formation within Human Neutrophils Purified human blood neutrophils $\left(10^{6}\right.$ cells $/ \mathrm{ml}$ of $\left.\mathrm{HBSS}\right)$ were stimulated with LPS $(500 \mathrm{ng} / \mathrm{ml})$ or vehicle (HBSS) at $37^{\circ} \mathrm{C}$ for $1 \mathrm{~h}$. The leukocytes were pretreated with $N$. procerum LAE $(0.2 \mu \mathrm{g} / \mathrm{ml})$ for 15 min before stimulation. After stimulation, cells were prepared for lipid body evaluation, as described above.

Statistical Analysis Results were represented as mean \pm standard error of mean (S.E.M.). The statistical analysis involving two groups was done using Student's $t$ test. ANOVA followed by Neuman-Keuls Student test was used to compare three or more groups. The results were considered significant with values of $p<0.05$.

\section{RESULTS}

N. procerum LAE Inhibits LPS-Induced Neutrophil 
Migration and PGE, Production in Vivo As previously demonstrated, ${ }^{5,12}$ the injection of LPS (250 ng/cavity) induced rapid influx of neutrophils as noted $6 \mathrm{~h}$ after LPS stimulation. Confirming our previous results, ${ }^{1)}$ pre-treatment with $N$. procerum LAE $(0.2,2,20 \mathrm{mg} / \mathrm{kg}$, i.p.) inhibited in a dosedependent manner the neutrophil accumulation induced by LPS (Fig. 1). As shown in Fig. 1B, in vivo LPS stimulation also triggers $\mathrm{PGE}_{2}$ production within $6 \mathrm{~h}$. Consistent with $N$. procerum LAE ability to inhibit cyclooxygenase-2 (COX-2) in vitro, ${ }^{1)}$ here we demonstrate that in vivo LPS-induced pleural $\mathrm{PGE}_{2}$ production was also sensitive to $N$. procerum LAE pre-treatment in a dose-dependent manner.

$N$. procerum LAE Potently Inhibits LPS-Induced Lipid Body Formation within Pleural Leukocytes in Vivo Lipid bodies are sites for compartmentalized eicosanoid formation that increase in numbers within leukocytes after different inflammatory insults including LPS stimulation. ${ }^{8)}$ Increased lipid body numbers within a recruited cell can be used as a marker of leukocyte activation. As shown in Fig. 2, LPS injection induced an increase in the numbers of lipid bodies within recruited leukocytes (mostly neutrophils) in the pleural cavity within $6 \mathrm{~h}$. Pre-treatment with different concentrations of $N$. procerum LAE $(0.2,2,20 \mathrm{mg} / \mathrm{kg}$; Fig. $2 \mathrm{~A})$ or ASA $(100,200,500 \mathrm{mg} / \mathrm{kg}$; Fig. $2 \mathrm{~B})$ was able to reduce lipid body numbers found within LPS-stimulated mice pleural leukocytes.

N. procerum LAE Inhibits the LPS-Induced Production of Inflammatory Cytokines in Vivo It is well known that LPS triggers inflammation in part by inducing release of cytokines from inflammatory cells, such as macrophages bearing Toll like receptor-4 (TLR-4). ${ }^{13)}$ In order to evaluate the mechanisms involved in $N$. procerum LAE ability to impair LPS-induced neutrophilia, we investigated whether this extract was able to affect cytokine production on LPS-induced pleurisy. As shown in Fig. 3, LPS induced both IL-1 and IL-6 release in mice pleural fluid $6 \mathrm{~h}$ after i.t. injection. Pre-treatment with $N$. procerum LAE $(2 \mathrm{mg} / \mathrm{kg})$ significantly decreased the levels of IL-1 (Fig. 3A) and IL-6 (Fig. 3B) recovered from the pleural wash of LPS-stimulated animals. In contrast, LPS-induced secretion of these cytokines (IL-1 and IL-6) and others (IL-10, KC and tumor necrosis factor (TNF)) in vitro by cultured mice peritoneal macrophages was not affected by $N$. procerum LAE, indicating that the cellular target of $N$. procerum LAE responsible in vivo for cytokine production may not be the resident macrophages, but possibly migrating cells. Of note, although $N$. procerum LAE failed to inhibit macrophage cytokine release, these cells are sensitive to $N$. procerum LAE. As shown in Table 1, N. procerum LAE significantly inhibited LPS-induced formation of new lipid bodies within macrophages, but did not alter the basal numbers of lipid bodies found within non-stimulated macrophages (data not shown).

PAF- and fMLP-Induced Pleural Neutrophil Accumulation Were Sensitive to Inhibition by $N$. procerum $\mathrm{LAE}$ PAF and fMLP were described as important inductors of in vivo neutrophilic reaction, including mice pleurisies. ${ }^{6,14}$ As shown in Fig. 4, N. procerum LAE $(2 \mathrm{mg} / \mathrm{kg})$ abolished both fMLP- and PAF-induced neutrophil accumulation in the mouse pleural cavity noted within $6 \mathrm{~h}$ (Figs. 4A, B, respectively).

N. procerum LAE Inhibits Human Neutrophil Function
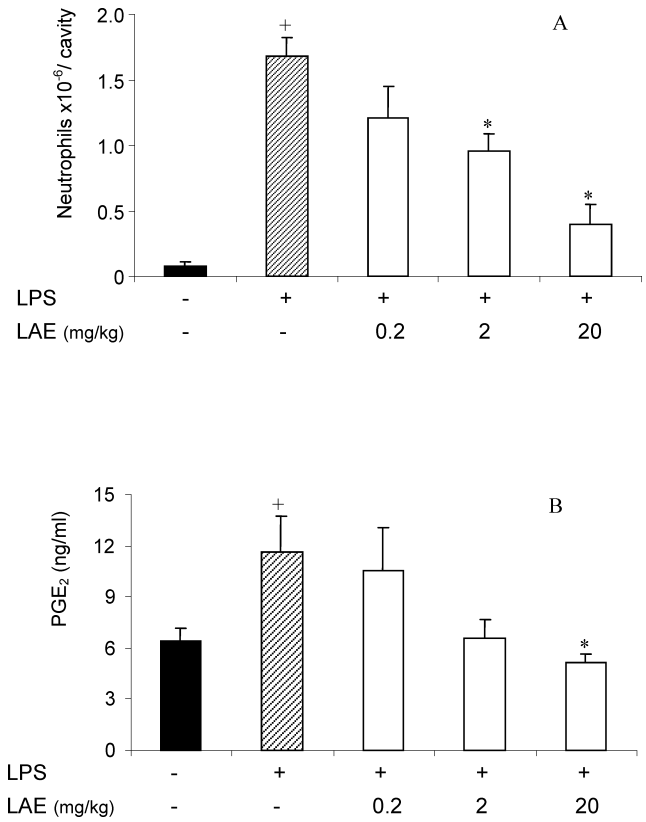

Fig. 1. N. procerum LAE Inhibits LPS-Induced Neutrophil Accumulation Associated to $\mathrm{PGE}_{2}$ Inhibition in the Mice Pleural Cavity

Neutrophil counts (A) or $\mathrm{PGE}_{2}$ quantification (B) were performed in the pleural wash $6 \mathrm{~h}$ after the i.t. injection of LPS $(250 \mathrm{ng} /$ cavity). LAE $(0.2,2,20 \mathrm{mg} / \mathrm{kg})$ was administered i.p. $1 \mathrm{~h}$ before LPS injection. Control animals received i.p. injection of saline. Sal represents animals that received an intrathoracic injection of saline. Each bar is the mean \pm S.E.M. from at least 8 animals, $* p<0.05$ when compared to control and $+p<0.05$ when compared to saline treated animals (ANOVA Student's Newman Keuls multiple comparisons test and Kruskal-Wallis test).
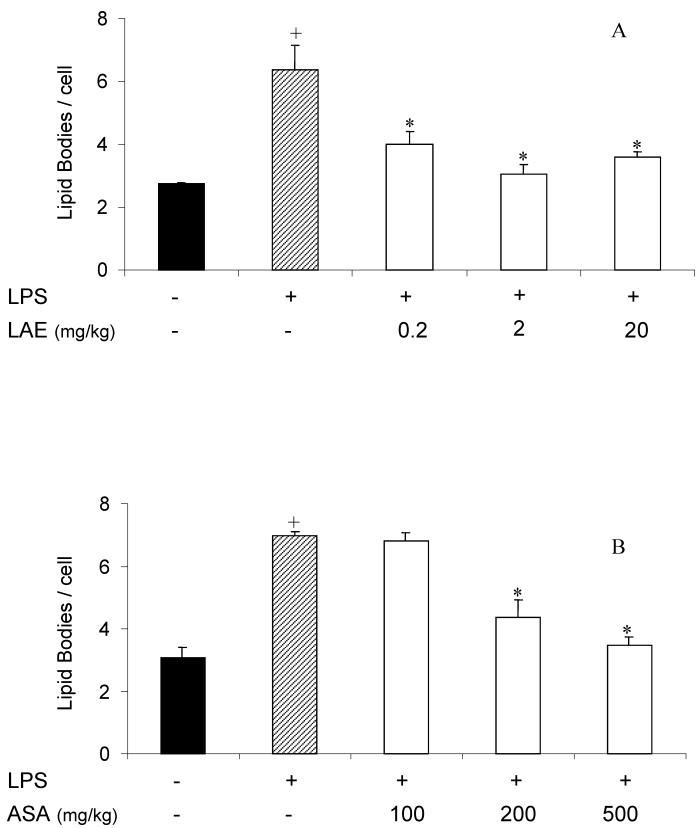

Fig. 2. N. procerum LAE Inhibits LPS-Induced Lipid Body Formation in Mouse Pleural Leukocytes

Lipid body enumeration were performed in leukocytes from the pleural wash $6 \mathrm{~h}$ after the i.t. injection of LPS (250 ng/cavity). In (A) LAE $(0.2,2,20 \mathrm{mg} / \mathrm{kg})$ or in (B) ASA $(100,200,500 \mathrm{mg} / \mathrm{kg})$ were administered i.p. $1 \mathrm{~h}$ before LPS injection. Control animals received i.p. injection of saline. Sal represents animals that received an intrathoracic injection of saline. Each bar is the mean \pm S.E.M. from at least 8 animals, $* p<0.05$ when compared to control and $+p<0.05$ when compared to saline treated animals (ANOVA Student's Newman Keuls multiple comparisons test).

in Vitro In addition to inhibiting neutrophil accumulation in vivo, $N$. procerum LAE also demonstrated a potent inhibitory effect on human neutrophil chemotactic activity in 
Table 1. In Vitro Effects of N. procerum LAE on Activation of Mouse Macrophages

\begin{tabular}{lcccccc}
\hline \hline \multirow{2}{*}{ Condition } & \multicolumn{5}{c}{ Cytokines (ng/ml) } & Lipid bodies/macrophage \\
\cline { 2 - 6 } & IL-10 & IL-1 & KC & TNF & IL-6 \\
\hline PBS & $0.1 \pm 0.1$ & $0.00 \pm 0.00$ & $8.6 \pm 0.7$ & $0.2 \pm 0.1$ & $0.9 \pm 0.4$ & $3.7 \pm 0.2$ \\
LPS & $0.8 \pm 0.1^{+}$ & $0.05 \pm 0.01^{+}$ & $322.9 \pm 28.5^{+}$ & $1.3 \pm 0.4^{+}$ & $2.9^{+} \pm 0.2^{+}$ & $8.2^{+} \pm .5^{*}$ \\
LPS+LAE $(0.2 \mu \mathrm{g} / \mathrm{ml})$ & $0.7 \pm 0.1$ & $0.06 \pm 0.02$ & $602.3 \pm 53.2$ & $1.2 \pm 0.3$ & $2.8 \pm 0.2$ & $5.6 \pm 0.3^{*}$ \\
LPS $+\mathrm{LAE}(2 \mu \mathrm{g} / \mathrm{ml})$ & $0.7 \pm 0.1$ & $0.04 \pm 0.01$ & $290.3 \pm 25.6$ & $1.2 \pm 0.3$ & $2.8 \pm 0.2$ & $5.0 \pm 0.3^{*}$ \\
\hline
\end{tabular}

$N$. procerum LAE $(2,0.2 \mu \mathrm{g} / \mathrm{ml})$ were incubated with mouse peritoneal macrophages for $15 \mathrm{~min}$ before LPS (500 ng/ml) stimulation. Lipid body formation within macrophages was evaluated $1 \mathrm{~h}$ after stimulation whereas cytokine release was evaluated after $6 \mathrm{~h}$. Lipid body numbers represent the means \pm S.E.M. of 50 macrophages recovered from at least 5 mice. $+p \leq 0.001$ compared to PBS group. $* p \leq 0.001$ compared to stimulated non-treated groups.
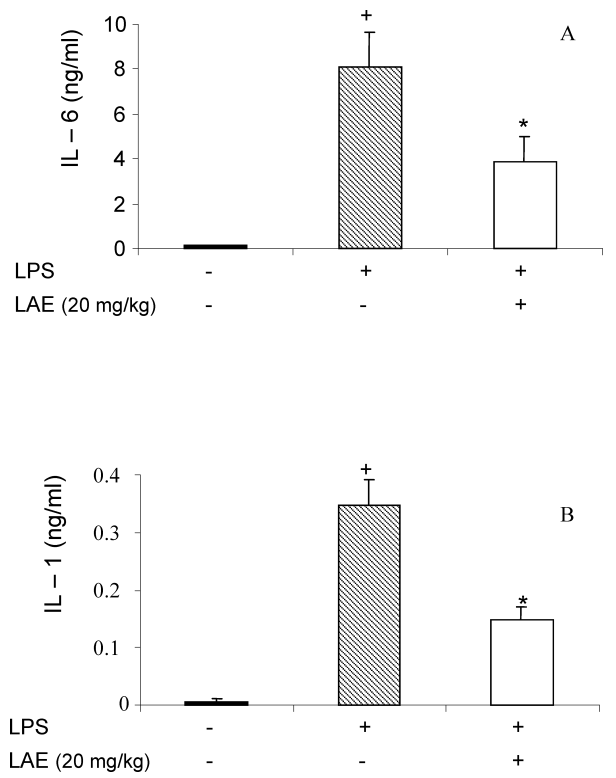

Fig. 3. N. procerum LAE Inhibits LPS-Induced IL-1 and IL-6 Accumulation in the Mouse Pleural Cavity

IL-1 (A) or IL-6 (B) amounts were quantified by ELISA in the pleural wash $6 \mathrm{~h}$ after the i.t. injection of LPS $(250 \mathrm{ng} /$ cavity). LAE $(20 \mathrm{mg} / \mathrm{kg})$ was administered i.p. $1 \mathrm{~h}$ before LPS injection. Control animals received i.p. injection of saline. Sal represents animals that received an intrathoracic injection of saline. Each bar is the mean \pm S.E.M. from at least 8 animals, $* p<0.05$ when compared to control and $+p<0.05$ when compared to saline treated animals (ANOVA Student's Newman Keuls multiple comparisons test).

vitro. PAF is a potent chemotactic agent for human neutrophils in vitro. ${ }^{11)}$ As shown in Fig. 5, PAF $\left(10^{-6} \mathrm{M}\right)$ induced an important chemotactic effect on human neutrophils assayed in a Boyden chamber. Pre-incubation of neutrophils with $N$. procerum LAE $(0.01 \mathrm{mg} / \mathrm{ml})$ completely inhibited PAF-induced neutrophil chemotaxis, without affecting neutrophil chemokinesis since $N$. procerum LAE did not modify random migration of non-stimulated neutrophils (Fig. 5). In addition, as shown in Table 2, N. procerum LAE blocked formation of new cytoplasmic lipid bodies within human neutrophils induced in vitro by PAF or LPS, suggesting that $N$. procerum LAE targets not only the locomotory machinery of neutrophils, but also a more critical step of neutrophil activation. Of note, basal numbers of cytoplasmic lipid bodies found within non-stimulated neutrophils were not modified by $N$. procerum LAE treatment (Table 2 ).

\section{DISCUSSION}

The aqueous crude extract prepared from the leaves of the
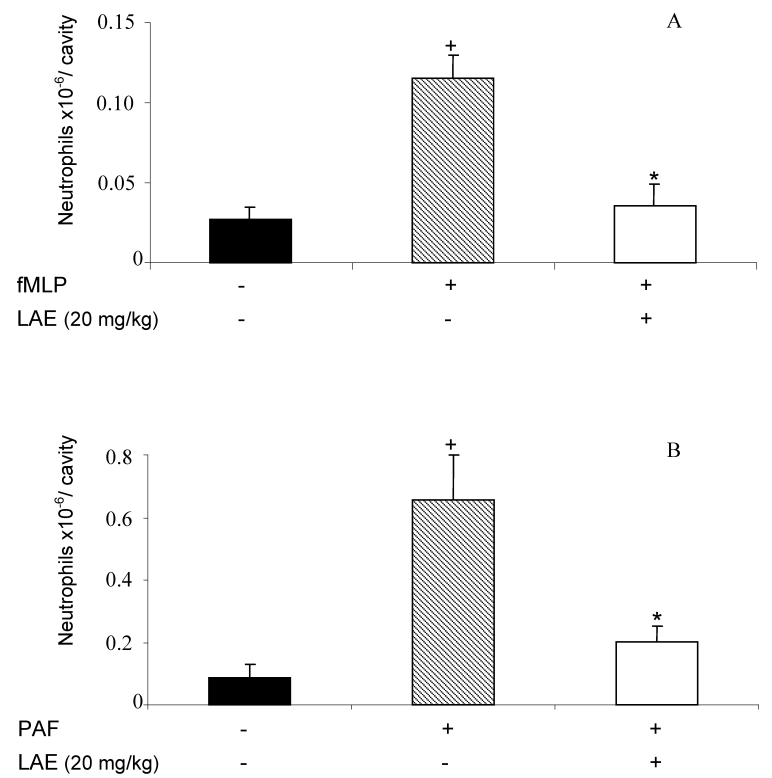

Fig. 4. N. procerum LAE Inhibits Both fMLP- and PAF-Induced Neutrophil Accumulation in the Mouse Pleural Cavity

Neutrophil counts were performed in the pleural wash $6 \mathrm{~h}$ after the i.t. injections of fMLP (200 ng/cavity, in (A)) or PAF (500 ng/cavity, in (B)). LAE $(20 \mathrm{mg} / \mathrm{kg})$ was administered i.p. $1 \mathrm{~h}$ before fMLP or PAF injections. Control animals received i.p. injection of saline. Sal represents animals that received an intrathoracic injection of saline. Each bar is the mean \pm S.E.M. from at least 8 animals, $* p<0.05$ when compared to control and $+p<0.05$ when compared to saline treated animals (ANOVA Student's Newman Keuls multiple comparisons test).

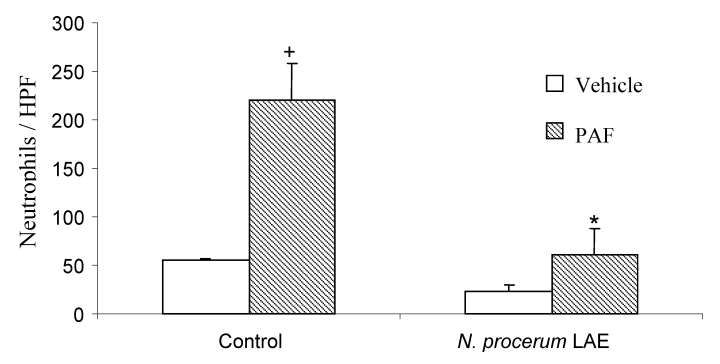

Fig. 5. N. procerum LAE Inhibits PAF-Induced Human Neutrophil Chemotaxis in Vitro

Human neutrophils were pre-incubated with vehicle or $N$. procerum LAE $(0.01$ $\mathrm{mg} / \mathrm{ml}$ ). Fifteen minutes after incubation, cells were stimulated with saline (open bars) or PAF $\left(10^{-6} \mathrm{M}\right.$, hatched bars). Each bar is the mean \pm S.E.M. from at least $2.5 \times 10^{5}$ cells obtained from 3 different human donors. The results are expressed in cells per high power field (HPF), $* p<0.05$ when compared to control group PAF and $+p<0.05$ when compared to vehicle (ANOVA Student's Newman Keuls multiple comparisons test). 
Table 2. In Vitro Effect of $N$. procerum LAE on Activation of Human Neutrophils, as Assessed by Induction of Lipid Body Formation

\begin{tabular}{lcc}
\hline \hline Stimuli & Treatment & Lipid bodies/neutrophil \\
\hline Control & - & $5.2 \pm 0.4$ \\
Control & LAE & $6.4 \pm 0.7$ \\
LPS & - & $9.4 \pm 0.4^{+}$ \\
LPS & LAE & $5.0 \pm 0.4^{*}$ \\
PAF & - & $10.5 \pm 0.7^{+}$ \\
PAF & LAE & $5.1 \pm 0.3^{*}$
\end{tabular}

$N$. procerum LAE $(0.2 \mu \mathrm{g} / \mathrm{ml})$ were incubated with human neutrophils for $15 \mathrm{~min}$ before PAF $(1 \mu \mathrm{M})$ or LPS $(500 \mathrm{ng} / \mathrm{ml})$ stimulation. Lipid body formation within neutrophils was evaluated $1 \mathrm{~h}$ after stimulation. This is a representative experiment where values represent the means \pm S.E.M. of 50 human neutrophils. $+p \leq 0.001$ compared PBS group. $* p \leq 0.001$ compared to stimulated non-treated groups.

bromeliad Nidularium procerum was shown to have a potent analgesic and anti-inflammatory activity in surrogate models of pain and inflammation. ${ }^{1)}$ The results obtained in the present study confirm the potent anti-inflammatory effects on LPS-induced neutrophilic reaction and reveal the possible mechanisms responsible for this effect.

Bacterial lipopolysaccharide (LPS) is an active fragment of the outer membrane of Gram-negative bacteria that has been used as an important tool to study the physiopathology of the inflammatory process. Neutrophil migration is a hallmark of the acute inflammatory process and is specially prominent after LPS challenge. ${ }^{5,12,15,16)}$ Following recognition by TLR4 and activation of TLR signaling pathways, LPS induces not only the production of prostanoids but also the production and release of an array of cytokines that are essential for the development of the subsequent inflammatory process. ${ }^{17)}$ The pro-inflammatory cytokines, TNF, IL-1, IL-6, Macrophage inhibitory protein- $1 \alpha$ MIP- $1 \alpha$, and MIP$1 \beta$ play pivotal roles in stimulating the early stages of acute inflammation, including the recruitment and activation of inflammatory cells like neutrophils, stimulation of endothelial cell activation, and direct cytotoxicity. ${ }^{18-21)}$ The finding that $N$. procerum LAE dose-dependently inhibits LPS-induced neutrophil accumulation in the mouse pleural cavity, reinforces our previous results ${ }^{1)}$ and clearly demonstrates the anti-inflammatory activity of this extract. The multistep process underlying the mechanisms involved in the development of endotoxin triggered tissue neutrophilia depends on at least three combined cellular components: (i) resident cells that primarily recognize the LPS; (ii) the chemoattractants/cytokines produced in situ (e.g., PAF, MIP-1 $\alpha$ and $\mathrm{KC}$ ); and (iii) the transmigrating and activated neutrophils themselves. When administered to LPS-stimulated mice, $N$. procerum LAE inhibited pleural neutrophilia and activation by a mechanism that appeared to depend on local events of endotoxemic inflammatory response, such as production of cytokines/chemo-attractants and/or function of transmigrating neutrophils.

The ability of $N$. procerum LAE to inhibit neutrophil accumulation can be partially explained by a mechanism dependent on $N$. procerum LAE-driven inhibition of LPS-induced in situ production of pro-inflammatory cytokines, including IL-1 and IL-6. Although production of chemokines is impaired by $N$. procerum LAE treatment, its cell target still needs to be defined since macrophages the most probable cell candidate do not appear to be the cytokine-producing cell sensitive to $N$. procerum LAE. The secretion of a variety of cytokines, including IL-1, IL-6, KC, IL-10 and TNF, by mouse macrophages stimulated in vitro with LPS was not modified by $N$. procerum LAE treatment. Different pleural resident cells of LPS-stimulated mice, such as mast cells or mesothelial cells, could represent the targets of $N$. procerum LAE.

We further investigated potential direct effects on neutrophils by evaluating $N$. procerum LAE effects on in vitro locomotory activity of neutrophils induced by PAF and fMLP. It is well known that local PAF action is strongly implicated in the early tissue neutrophil accumulation induced by $\mathrm{LPS}^{5)}$; PAF and PAF related molecules are able to directly attract neutrophils to the site of inflammation, ${ }^{14)}$ although evidence exists that this effect is dependent on the generation of leukotrienes in vivo. ${ }^{9,22,23)}$ On the other hand, fMLP unlike LPS and PAF, recruits neutrophils to inflamed tissues by a direct mechanism independent of lipid mediator and protein synthesis. $^{15,24)} N$. procerum LAE inhibited both in vitro chemotaxis and in vivo pleural recruitment of neutrophils induced by PAF or fMLP. Therefore, the $N$. procerum LAE actual cell target within endotoxemic inflammation appears to be the neutrophils themselves, which have their motility machinery disrupted. It is noteworthy that $N$. procerum LAE also inhibit an other important inflammatory function of neutrophils the formation of new cytoplasmic lipid bodies indicating that the $N$. procerum LAE intracellular target within neutrophils is an upstream step common to cell motility and lipid body genesis.

Lipid bodies are non-membrane-bound, lipid-rich cytoplasmic domains that characteristically increase in number within leukocytes associated with the inflammatory process. ${ }^{25}$ ) For instance, lipid body numbers are increased in eosinophils from patients with hypereosinophilic syndrome, ${ }^{26)}$ in leukocytes from inflammatory arthritis ${ }^{25)}$ and sepsis, ${ }^{8)}$ and from bronchoalveolar lavage of patients with acute respiratory distress syndrome, ${ }^{27,28)}$ Lipid bodies are candidates to play a major role in the formation of eicosanoid mediators during inflammation as they are sites of localization of several enzymes related to eicosanoid metabolism including COX-2, 5lipoxygenase, phospholipase $\mathrm{A} 2$, mitogen-activated protein kinase and phosphatidylinositide 3-kinase. ${ }^{25,29-31)}$ In addition, agents that inhibit lipid body formation in vitro inhibited the priming response for enhanced eicosanoid release. ${ }^{14)}$ Also yangambin, a competitive PAF receptor antagonist extracted from the Brazilian plant Ocotea duckei VATTIMO, is able to inhibit PAF-induced lipid body formation in mice peritoneal macrophages. ${ }^{32)}$ The inhibition of lipid body formation has been proposed as a novel strategy for anti-inflammatory therapies. ${ }^{25,28)}$ In this respect, ASA and other nonsteroidal anti-inflammatory drugs (NSAIDs) were described as effective inhibitors of leukocyte lipid body formation, ${ }^{25,28)}$ and this effect was shown to be independent of cyclooxygenase inhibition. ${ }^{25,28)}$ Moreover, PAF and cis-fatty acids stimulate numbers of lipid bodies correlated with enhanced levels of leukotrienes and prostaglandins in human PMNs and eosinophils. ASA and various NSAIDs inhibit lipid body formation and also inhibit the enhanced formation of lipoxigenase- and cyclooxygenase-derived eicosanoids. ${ }^{25)}$ Here, we showed that $N$. procerum LAE is able to block LPS-induced lipid body formation both in vitro and in vivo within neu- 
trophils. Moreover, LPS-induced in vivo production of $\mathrm{PGE}_{2}$ was decreased by $N$. procerum LAE treatment, suggesting that inhibition of lipid body formation may affect the production of prostanoids after LPS stimulation. Nevertheless, LPS is a potent stimulus for COX-2 expression ${ }^{33)}$ and one must take into account that $N$. procerum LAE has been previously shown to selectively inhibit COX-2 activity in an in vitro assay using cells overexpressing COX-2, ${ }^{1}$, which could explain the reduction in $\mathrm{PGE}_{2}$ production. In fact, our experiments show that doses of $N$. procerum LAE able to completely inhibit lipid bodies formation are not enough to significantly reduce $\mathrm{PGE}_{2}$ production, indicating that an additional effect might take place in parallel to inhibition of lipid body formation to reduce the formation of prostanoids.

Inasmuch as neutrophils are implicated as the major players of endotoxemic disorders, our results point toward the Brazilian bromeliaceae $N$. procerum as a phyto-pharmacological candidate for novel alternative anti-inflammatory therapies. $N$. procerum LAE exerts its anti-neutrophilic effects by interfering at different levels with the capacity of the host to respond to injury caused by endotoxin. Specifically, the mechanism of $N$. procerum LAE-driven inhibition of endotoxin-induced neutrophilia involves the inhibition of at least 3 key events: local cytokine production, neutrophil migratory activity and neutrophil activation.

Acknowledgments Thanks are due to Dr. Marco Aurélio Martins for helpful comments on the work. Rafaela Cordeiro Ré de Paiva, Leandro Pereira Costa and Edson F. Assis for the technical assistance. Dionísio Pessamilho, Director of Reserva Biológia Nacional de Poço das Antas/IBAMA, for the supply of plant material. Financial support of FAPERJ, CNPq and PAPES-Fiocruz. PTB is an International Research Scholar from Howard Hughes Medical Institute.

\section{REFERENCES}

1) Amendoeira F. C., Frutuoso V. S., Chedier L. M., Figueiredo M. R., Kaplan M. A. C., Bozza P. T., Castro-Faria-Neto H. C., Phytomedicine, 12, 78-87 (2005).

2) Chedier L. M., Kaplan M. A. C., Bromelia, 3, 25-31 (1996).

3) Fontoura T., Costa A., Wendt T., Selbyana, 12, 5- 45 (1991).

4) Leme E. M. C., "Nidularium," Sextante, Rio de Janeiro, 2000.

5) Bozza P. T., Castro-Faria-Neto H. C., Silva A R., Larangeira A. P., Silva P. M., Martins M. A., Cordeiro R. S., Eur. J. Pharmacol., 270 143-149 (1994).

6) Secco D. D., Paron J. A., de Oliveira S. H., Ferreira S. H., Silva J. S., Cunha Fde. Q., Nitric Oxide, 9, 153-164 (2003).

7) Castro-Faria-Neto H. C., Araujo C. V., Moreira S., Bozza P. T.,
Thomas G., Barbosa-Filho J. M., Cordeiro R. S., Tibirica E. V., Planta Med., 61, 106-112 (1995).

8) Pacheco P., Bozza F. A., Gomes R. N., Bozza M., Weller P. F., CastroFaria-Neto H. C., Bozza P. T., J. Immunol., 169, 6498-6506 (2002).

9) Silva A. R., de Assis E. F., Caiado L. F., Marathe G. K., Bozza M. T., McIntyre T. M., Zimmerman G. A., Prescott S. M., Bozza P. T., Castro-Faria-Neto H. C., J. Immunol., 168, 4112-4120 (2002).

10) Richards K. L., McCullough J., Immunol. Commun., 13, 49-62 (1984).

11) Alves A. C., Pires A. L., Cruz H. N., Serra M. F., Diaz B. L., Cordeiro R. S., Lagente V., Martins M. A., Eur. J. Pharmacol., 312, 89-96 (1996).

12) Larangeira A. P., Silva A. R., Gomes R. N., Penido C., Henriques M. G., Castro-Faria-Neto H. C., Bozza P. T., Inflamm. Res., 50, 309 (2001).

13) Fan H., Cook J. A., J. Endotoxin. Res., 10, 71-84 (2004).

14) Bozza P. T., Payne J. L., Goulet J. L., Weller P. F., J. Exp. Med., 183, $1515-1525$ (1996).

15) Cybulsky M. I., McComb D. J., Movat H. Z. Am. J. Pathol., 135, 227-237 (1989).

16) Ulich T. R., del Castillo J., Ni R. X., Bikhazi N., J. Leukoc. Biol., 45, 546-557 (1989)

17) Guha M., Mackman N., Cell Signal, 13, 85 (2001).

18) Dinarello C. A., Blood, 87, 2095-2147 (1996).

19) Wolpe S. D., Davatelis G., Sherry B., Beutler B., Hesse D. G., Nguyen H. T., Moldawer L. L., Nathan C. F., Lowry S. F., Cerami A., J. Exp. Med., 167, 570-581 (1988).

20) Wolpe S. D., Sherry B., Juers D., Davatelis G., Yurt R. W., Cerami A., Proc. Natl. Acad. Sci. U.S.A., 86, 612-616 (1989).

21) Zhang M., Borovikova L. V., Wang H., Metz C., Tracey K. J., Mol. Med., 5, 595-605 (1999).

22) Silva P. M., Martins M. A., Castro-Faria-Neto H. C., Cordeiro R. S., Vargaftig B. B., Braz. J. Med. Biol. Res., 21, 855-858 (1988).

23) Weller P. F., Bozza P. T., Yu W., Dvorak A. M., Int. Arch. Allergy Immunol., 118, 450—452 (1999).

24) Thomazzi S. M., Souza M. H., Melo-Filho A. A., Hewlett E. L., Lima A. A., Ribeiro R. A., Braz. J. Med. Biol. Res., 28, 120-124 (1995).

25) Bozza P. T., Payne J. L., Morham S. G., Langenbach R., Smithies O., Weller P. F., Proc. Natl. Acad. Sci. U.S.A., 93, 11091-11096 (1996).

26) Bozza P. T., Yu W., Cassara J., Weller P. F., J. Leukoc. Biol., 64, 563569 (1998).

27) Triggiani M., Oriente A., Seeds M. C., Bass D. A., Marone G., Chilton F. H., J. Exp. Med., 182, 1181-1190 (1995).

28) Bozza P. T., Pacheco P., Yu W., Weller P. F., Prostaglandins Leukot. Essent. Fatty Acids, 67, 237-244 (2002).

29) Bandeira-Melo C., Bozza P. T., Weller P. F., J. Allergy Clin. Immunol., 109, 393-400 (2002).

30) Yu W., Bozza P. T., Tzizik D. M., Gray J. P., Cassara J., Dvorak A. M., Weller P. F., Am. J. Pathol., 152, $759-769$ (1998).

31) Yu W., Cassara J., Weller P. F., Blood, 95, 1078-1085 (2000).

32) Jesus-Morais C. M., Assis E. F., Cordeiro R. S., Barbosa-Filho J. M., Lima W. T., Silva Z. L., Bozza P. T., Castro-Faria-Neto H. C., Planta Med., 66, 211-216 (2000).

33) Maloney C. G., Kutchera W. A., Albertine K. H., McIntyre T. M., Prescott S. M., Zimmerman G. A., J. Immunol., 160, 1402-1410 (1998). 